\title{
Web Services Selection for Distributed Composition of Multimedia Content
}

\author{
Matthias Wagner \\ Future Networking Lab \\ DoCoMo Communications Laboratories Europe \\ Munich, Germany \\ wagner@docomolab-euro.com
}

\author{
Wolfgang Kellerer \\ Future Networking Lab \\ DoCoMo Communications Laboratories Europe \\ Munich, Germany \\ kellerer@docomolab-euro.com
}

\begin{abstract}
Growing numbers of pervasive devices are gaining access to the Internet. However, much of the existing rich multimedia content cannot be handled by mobile client devices with limited communication, processing, storage and display capabilities. In this paper, we propose new ways to enhance the universal access to multimedia content through Web Services and Semantic Web concepts. A semantic-based personalized delivery concept is drafted that makes use of these emerging technologies together with rather classical multimedia transcoding ideas. Instead of large, monolithic portal applications designed for multi-purpose adaptation and a single-source delivery, we propose to shift multimedia adaptation functionality to a portfolio of adequately selected Web Services. Web Services accessible through standard interfaces that allow for multimedia format conversion and composition can allow for a more flexible, application-independent adaptation and thus ease multimedia service provisioning essentially.
\end{abstract}

\section{Categories and Subject Descriptors}

H.3.5 [Information Storage and Retrieval]: Online Information Services - Web-based services.

\section{General Terms}

Algorithms, Languages, Design.

\section{Keywords}

Web Services, Semantic Web, Multimedia, Service Composition.

\section{INTRODUCTION}

The rising role of the Internet in providing multimedia applications opens up new challenges regarding flexibility and the availability of composable services. Already today, Internet portals, multimedia libraries or e-shops have to deliver combined content to support different kinds of users and different types of Web browsers. The need for personalized access to multimedia services is further accelerated by the vision of a future mobile Web with wireless access to content from anywhere, anytime. For instance, DoCoMo's m-stage service [8] was one of the first mobile services to deliver multimedia-type content in terms of streaming

Permission to make digital or hard copies of all or part of this work for personal or classroom use is granted without fee provided that copies are not made or distributed for profit or commercial advantage and that copies bear this notice and the full citation on the first page. To copy otherwise, or republish, to post on servers or to redistribute to lists, requires prior specific permission and/or a fee.

MM'04/, October 10-16, 2004, New York, New York, USA.

Copyright 2004 ACM 1-58113-893-8/04/0010 ...\$5.00 audio and is now being followed by similar services that provide video and other content to individual mobile users. Especially in the context of mobile Web access, emerging service paradigms such as Web Services are recognized as important building blocks. Web Services are Internet-based, distributed modular applications with precisely defined interfaces to enable flexible and effective service development and deployment. They promise to enable the new generation of Internet-based applications in supporting application-to-application as well as application-touser Internet communication. With Web Services, applications at different network locations can be integrated to function as if they were part of a single, large software system. Examples of applications made possible by Web services include automated business transactions and direct desktop/handheld device access to reservations, stock trading, and order-tracking systems.

In spite of its large momentum, the actual Web Service standardization is still mostly driven by a highly technical discussion: activation signatures, interface types, and quality of service characteristics are utilized to gain initial access to service subscriptions and subsequently allow service composing and the tailoring of service offerings. On the other hand, research in the area of the so-called Semantic Web is targeting at higher level of abstraction and semantics to enable truly intelligent services. Generally speaking, the Semantic Web encompasses efforts to populate the Web with content having formal semantics and rich service descriptions. Recent semantic efforts around UDDI, WSDL and SOAP try to enable automated agents to reason about Web service descriptions and to perform intelligent service discovery, comparison and composition [7]. In the following we present our vision of how to streamline the emerging technologies, Web Services and Semantic Web, together with multimedia delivery concepts to achieve a flexible, personalized selection of a Web services for the orchestrated composition of multimedia content.

\section{MULTIMEDIA SYNDICATION}

The need for personalized access to multimedia services is largely driven by the vision of a future mobile Web with wireless access to content from anywhere, anytime. Very often it is necessary to tailor multimedia data in a user- or device centered fashion for particular applications. Advanced content syndication and delivery concepts therefore have to regard service selection and composition based on large sets of possibly applicable services, context information and user preferences. The richer and the more complex multimedia content gets, the more a delivery service provider can benefit from a user-centric composition and syndication of content. As a consequence, adaptation and personalization 
questions become more and more crucial for successful service provisioning.

In the past, several methods for scalable and personalized composition as well as delivery of multimedia data have been put forward, e.g., [9], [11] or [12]. For instance, [11] proposes the InfoPyramid scheme to interrelate different formatting and conversion options of multimedia objects together with composition strategies for complex multimedia documents. As depicted in Figure 1, the InfoPyramid manages different modalities (video, image, text and audio) and fidelities (summarized, compressed or scaled variations) of a media object. Each object is represented by a cell of the pyramid. For example, in Figure 1, the cell in the lower left corner corresponds to a high-quality video encoding of a sample objects and acts as the source for other conversions. In [11] a subjective utility function is then introduced that assigns each InfoPyramid cell a value that reflects its utility (or information value) in a particular application context. Based on this utility function different optimization strategies can be defined that deal with the composition of complex multimedia documents from multiple InfoPyramids-objects under constraints, e.g., to maximize the combined utility of a document w.r.t. a given size constraint like the buffer size of a data receiving mobile client.

While frameworks like the InfoPyramid provide a good starting point for optimized multimedia delivery, we advocate that in complex mobile scenarios decentralized and semantically richer models are needed to best support mobile clients in a given situation or context. To flesh out this claim, Figure 2 illustrates a personalized delivery scenario that we will further on refer to: A smartphone user requests the latest news from a service provider. Available multimedia content includes a news ticker and topical videos available in MPEG 2 only. The news provider has no adaptation capabilities, so additional services are required to serve the user's request. For the delivery, the multimedia content requires transcoding to fit the target format, compression to adapt to the wireless link, and also text translation for the ticker. Furthermore, the limited smartphone display requires integrating the ticker with the video stream.

\section{WEB SERVICES FOR DISTRIBUTED MEDIA COMPOSITION}

Web Services are Internet-based, modular applications that provide standard interfaces and communication protocols aiming at loosely coupled service integration. Several key standards have emerged to define the advertisement, discovery, and usage of Web Services and to form the foundation for Web services: For

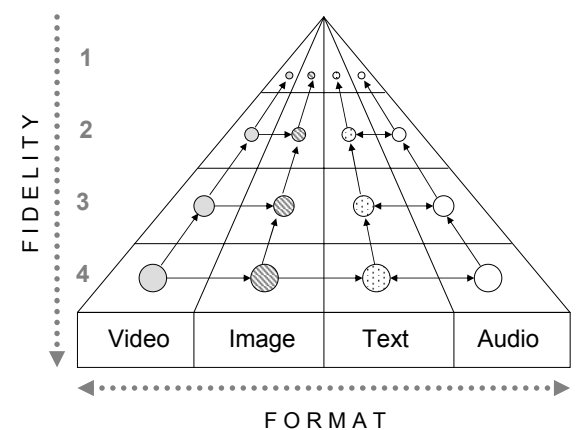

Figure 1: InfoPyramid -selection through format and fidelity.

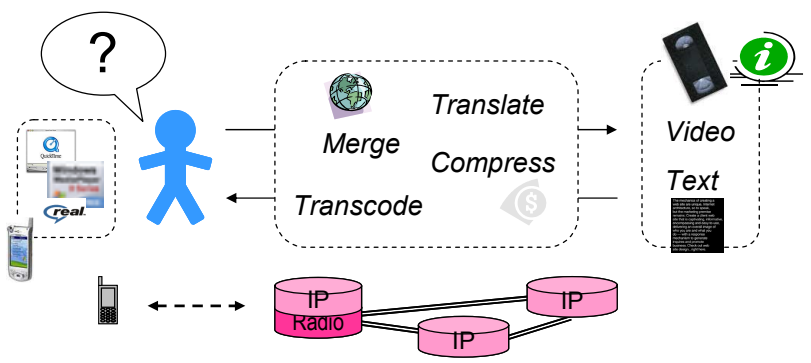

Figure 2: Multimedia Syndication Usage Scenario.

advertisement, service descriptions based on the Web Service Description Language (WSDL) [5] are made available on UDDI registries for Universal Description, Discovery and Integration [13]. WSDL and UDDI are complemented by SOAP, a protocol for information exchange. In addition, standards are underway to facilitate automated business process integration among trading partners, e.g., ebXML (Electronic Business XML). By supporting an advanced business model of service advertisements and composition, Web Services are considered to play an increasing role also for services beyond standard Web page information services. In particular, in today's distributed (wireless) networking environment, where systems have to dynamically react on frequent changes regarding transmission parameters (e.g., data rate, delay), device capabilities (e.g., display, battery) as well as user requirements (e.g., preferences), a more flexible system design is needed.

In contrast to conventional systems characterized by a monolithic structure or a set of components with proprietary interfaces, the use of Web Services offers the following advantages. Software is not only provided in a component oriented way, but as services with standardized interfaces to support many different applications. This reduces programming complexity in two ways. First, separate components make software updates or changes transparent to the application. Second, standardized interfaces allow third parties to contribute their services with low integration effort to applications. Revisiting our running example, Figure shows one possible composition of Web Services to achieve the user preferred syndication of media streams in our use case. Before a Merging Web Service performs the combination of ticker and video, the video stream is transcoded and the text is translated into the users preferred language. After merging, a compression component adapts the source rate to channel and device capabilities.

\section{TOWARDS SEMANTIC-BASED WEB SERVICES}

The (classic) content syndication concept described in Section 2, addresses the combination of different media representations such as text, audio, video, image available in different modalities and fidelity levels according to the constraints given by the respective service request. However, in our scenario such a selection is, in addition, subject to network capabilities as well as user preferences. Furthermore, the actual composition can benefit from services available in the network like third party transcoding or translation services. Thus, while the principal selection problem remains the same, additional complexity is added since we have to regard interdependencies between consecutive service components. Input and output conditions have to be met all along the service chain. Moreover, knowledge about the internal process of 


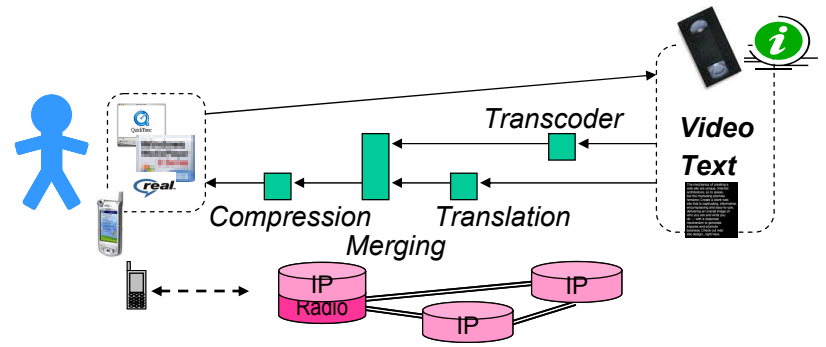

Figure 3: Media syndication through loosely coupled services

a complex service component that is composed of subcomponents also helps to select a service portfolio that is providing the functionality in a more meaningful way for the user.

The syndication illustrated in Figure 3 represents only one of possibly many options found through keyword matching in UDDI registries. Other solutions, e.g., having the Transcoder after the Merging, might be more efficient in respect with available resources. To find an optimal solution among many options, more information about the services is needed. Here, semantic information is of high value. Also, semantic information would support a relaxation of a search, i.e., to still find an acceptable solution if there is not direct match. However, the current Web Service standard such as UDDI is limited to exact keyword matching and does not support semantic matching in order to achieve a meaningful matching of service advertisements to service requests [4].

\section{SERVICE DISCOVERY, SELECTION AND COMPOSITION}

Strongly influenced by research on the Semantic Web, OWL-S is an ontology-based approach to the semantic description of Web Services [5]. The goal of OWL-S is to provide a service ontology that allows software agents to discover, execute and compose web services automatically. Referring to the Web Services standards as described above, OWL-S replaces the WSDL description. A registry such as UDDI or a communication protocol such as SOAP is not part of the OWL-S definition. Currently, the structure of the OWL-S ontology is threefold and consists of a service profile, a process model, and a service grounding. The service profile supports semantic-based service advertising and discovering. The process model gives a detailed description of a service's operation. The service grounding provides details on how to interoperate with a service via message exchange. Whereas the first two models serve as abstract Web Service specifications, the service grounding describes the communication with a concrete Web Service. This model directly corresponds to the WSDL description. In fact, OWL-S provides an ontology for grounding process descriptions to WSDL.

Let us study the benefits of using OWL-S in our running scenario. Ontologies represent elements of a domain together with their relationships. Thus, when searching for a suitable transcoder in our scenario we can make use of knowledge about the relationships of devices, video players, video encodings, transmission networks and their capabilities (e.g., support of codecs, data rates). Figure 4 shows an example ontology that partially models our domain of interest. Note that the highlighted taxonomy of Video formats can be interpreted as an orthogonal extension to the InfoPyramid scheme (cf. Section 2): while in the InfoPyramid different video formats and fidelities are only distinguished through utility values, the video ontology provides deeper insights into format interrelationships and the usage of these formats (in media players and devices). During service provisioning, OWL-S ontologies can serve as knowledge bases that are consulted if nontrivial decisions must be made, e.g., to check the compliance of a certain video encoding with the media player on the client side. In addition, search strategies in an ontology can be such that opposed to exactly matching features and capabilities, the search objective is gradually relaxed along the lines of the ontology to the nearest best service match [2][3].

The OWL-S process model furthermore allows describing a workflow, which is not possible with conventional Web Service descriptions. Two main advantages make this process model especially beneficial for the herein discussed service composition. First, we are able to view the internal process of one Web Service and use this information for a refined selection. Second, and most important, we can describe the process of a set of composed Web Services and thus represent this set as a Web Service itself. This allows easy reuse by leveraging from providing a separate implementation of a composed Web Service. The OWL-S description just serves as such (virtual) Web Service. In our example the Merging Web Service is composed of several services (Figure 5).

The provisioning of complex, user tailored services has changed the service usage flow from a simple service start - execution termination pattern. In order to provide the right service at the right time to the user phases prior to service execution such as service discovery, selection and composition have to get high attention. This brings us back to multimedia syndication using distributed services, which requires sophisticated mechanisms in service discovery, selection and composition. Solutions for discovery and selection of Web Services have already been described in [2] and [3], which show a basic approach for the discovery of a set of suitable services in a large service catalogue and the selection of one service that matches the detailed application requirements. The focus is rather on single Web Services; however the basic principles are also usable for service composition. The main motivation for the work in [2] and [3] was service personalization, i.e., supporting the user in searching for a Web Service. This requirement also applies for multimedia syndication. Here, the discovery and selection of a suitable service portfolio also does not only depend on technical constraints (so called hard constraints), but also on user-specific requirements, such as preferences. The latter requirements represent so called soft con-

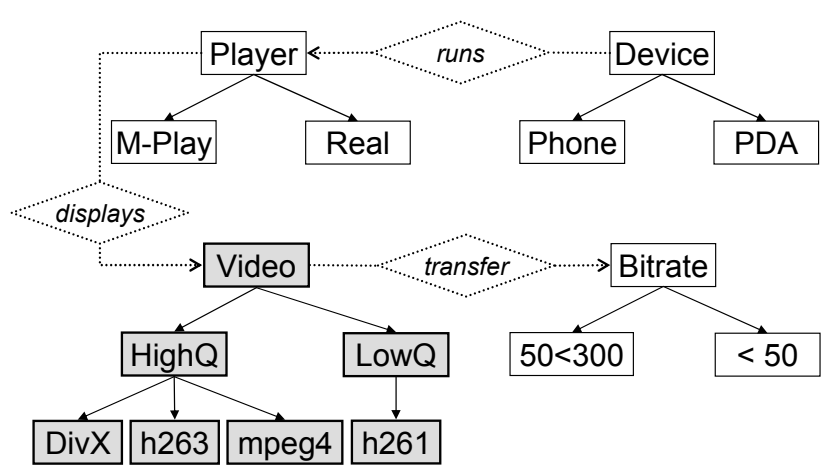

Figure 4: Sample ontology to express device and multimedia format dependencies. 


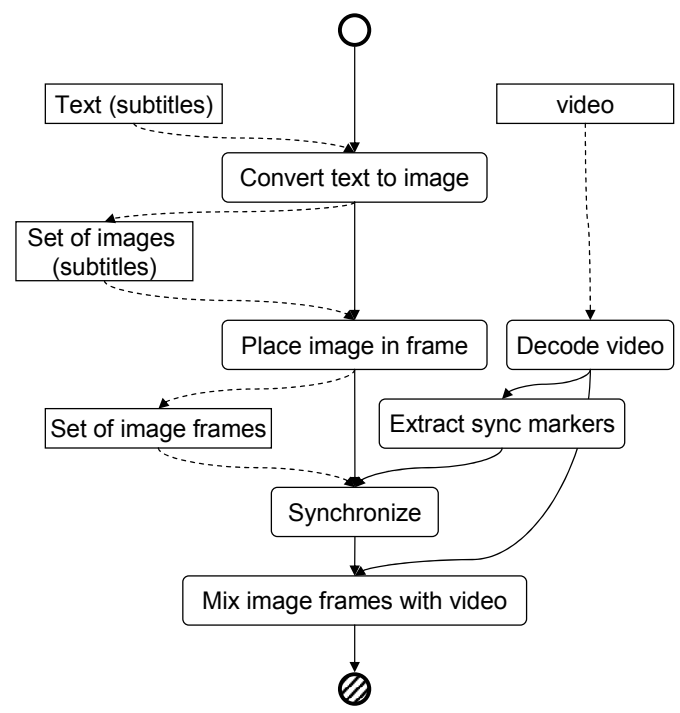

Figure 3: Merging of media object in a complex Web Service.

straints, in which a user expresses likes and dislikes, e.g., "I prefer a PDA over a mobile phone" or "I prefer M-player to Realplayer".

In [2] a detailed algorithm for the preference-based discovery of Web Services is described. Main point is a conceptualization of Web Services given as service ontology. This ontology serves as the service space with the default preferences shown by relationships (such as "is subset of") of the nodes representing a service category. An application supporting a search for a user can relax along the lines of this ontology to find an available web service for a user. In order to optimize the service discovery, specific user preferences are taken into account in addition. These preferences can be regarded as additional relationships between the nodes used for further relaxation.

\section{CONCLUSION AND OUTLOOK}

In this paper we have aimed at contributing to an emerging topic: the combination of multimedia communications and Web technologies (Web Services) together with aspects from the Semantic Web (OWL-S). Research in this area is just starting and in most cases only touches purely technical Web Services aspects. Based on the described mechanisms for the discovery and selection of single Web Services we have outlined how "semantic sugar" which is added by OWL-S can support a more meaningful Web Service provisioning. In addition, preferences support the shift from exact keyword matching in UDDI repositories to meaningful navigations and relaxations in service ontologies. [6] shows a concept that applies preferences to the selection of multimedia services in a SIP environment.

Furthermore, service composition, as needed for multimedia syndication, faces more complex challenges. Again a semantic-based description complementing the basic, conventional interface description of Web Services, support the chaining of services in order to select the optimal solution among a set of alternative service portfolios. In addition to the selection of a Web Service portfolio for composition, it is also necessary to be able to de- scribe the behavior of the portfolio. Here, process models do not only allow having a standardized workflow description, but also enable the reuse of service portfolios without hardcoding them. OWL-S as the most advanced description model for Web Services also provides such support for process descriptions.

In our view, the trend towards semantically enriched Web Service to open up new Web Service application domains can be considered significant. For instance, standardization activities in telecommunication industry deal with the provisioning of multimedia applications through Web Services. For instance, Parlay X [10] applies the Web Services paradigm and related standards to OSA/Parlay to allow for Internet access to telecommunication services. OSA/Parlay APIs are standardized, open interfaces for application providers to telecommunication network services such as call control, messaging, location information. Parlay X Services represent a simplified abstraction from the OSA/Parlay APIs which are merely independent component interfaces without explicit support for component distribution and composition. Semantic Web Service and multimedia concepts combined with technology such as Parlay $\mathrm{X}$ promise to leverage sophisticated multimedia communication services in the future.

\section{REFERENCES}

[1] A. Ankolekar. OWL-S: Semantic Markup for Web Services, 2003. http://www.daml.org/services/owl-s/1.0/owl-s.pdf

[2] W.-T. Balke, M. Wagner. Cooperative Discovery for Usercentered Web Service Provisioning. In Proc. of the First Int. Conf. on Web Services (ICSW'03), Las Vegas, USA, 2003.

[3] W.-T. Balke, M. Wagner. Towards Personalized Selection of Web Services. In Proc. of the $12^{\text {th }}$ Int. World Wide Web Conference (WWW 2003), Budapest, Hungary, 2003.

[4] S. Balzer, T. Liebig, M. Wagner. Pitfalls of OWL-S - A Practical Semantic Web Use Case. (under submission)

[5] E. Christensen, F. Curbera, G. Meredith, S. Weerawarana. Web Services Description Language (WSDL) 1.1., W3C, http://www.w3c.org/TR/wsdl.

[6] W. Kellerer, M. Wagner, W.-T. Balke, H. Schulzrinne, Preference-based Service Management for IP-Based Mobile Multimedia Signaling, ETT, Wiley, July 2004.

[7] S. McIlraith, T. Son, and H. Zeng: Semantic Web Services. In IEEE Journal on Intelligent Systems, IEEE, 2001.

[8] M-Stage. http://www.nttdocomo.com

[9] A. Ortega, et al. Soft Caching: Web cache management techniques for images. In WS on Multimedia Signal Processing, pp. 475-480, Princeton, NJ, June 1997, IEEE.

[10] Parlay Group. Parlay APIs 4.0 Parlay X Web Services White Paper, http://www.parlay.org

[11] J. R. Smith, R. Mohan, C.-S. Li. Scalable Multimedia Delivery for Pervasive Computing. In Proc. ACM Intl. Conf. on Multimedia (ACM-MM), Orlando, FL, November, 1999.

[12] J. R. Smith. Digital Libraries and the Internet. IEEE Communications Magazine, 73(1):92-99, January 1999.

[13] UDDI. Technical White Paper. http://www.uddi.org. 